\title{
Preliminary Experience with a Gastrointestinal Endoscope Using a White Light-Emitting Diode
}

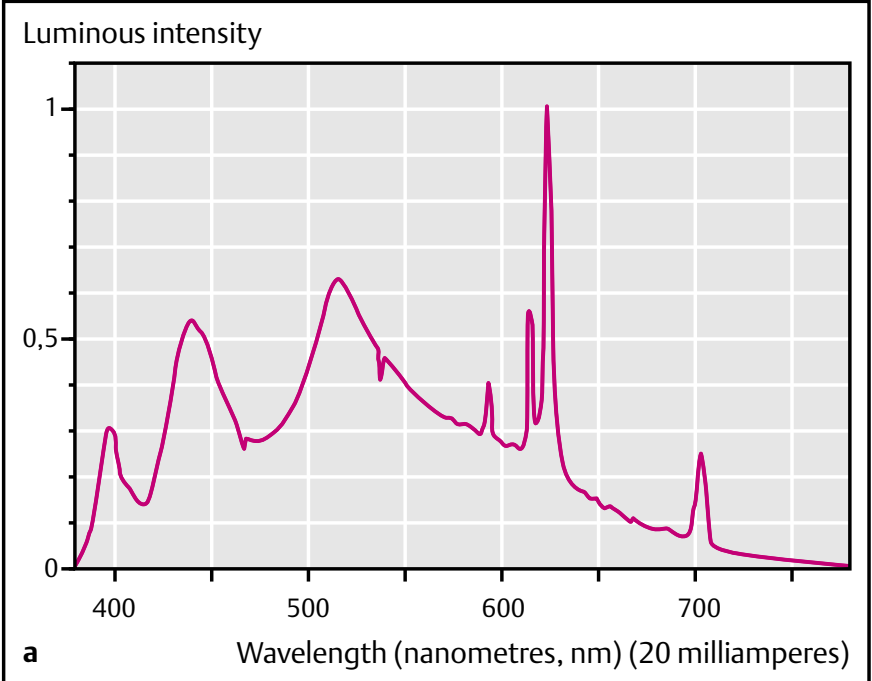

In the conventional video endoscope system, a large light source apparatus and light-guiding fibre bundle are essential [1]. The capsule endoscope is mainly useful for disease of the small intestine [2]. Recently, the light-emitting diode (LED) has been rapidly developed as a new compact, powerful light source [3]. We have developed an new experimental prototype LED-illuminated gastrointestinal endoscope.

For many conventional white LEDs, the peak luminous intensity is at a wavelength of 450 nanometers $(\mathrm{nm})$ in the blue range of the spectrum, and the color rendering in the clinically important red range at around $600 \mathrm{~nm}$ is thought to be insufficient. Our new white LED (developed by T. Taguchi) has its main peak of luminous intensity in the red range (Figure 1 a) [4]. It is a one-chip type orangeyellow-green-blue white LED based on a near-ultraviolet light-emitting diode with the highest luminous efficiency ( $43 \%)$. The prototype scope was mounted with two packages of 12 elements of the new white LED (Figure $\mathbf{1}$ b) (Fujinon Corporation, Saitama, Japan). We conducted esophagogastroduodenoscopy in the beagle, using painless procedures (at the Yamamoto Animal Hospital, Ube, Japan) with the approval of the institutional review board. The esophageal mucosa of
Figure 1 a The spectral characteristics of the new white light-emitting diode (LED) with the main peak of luminous intensity in the red range $(600 \mathrm{~nm})$. b Appearance of the prototype new white LED-illuminated endoscope (CCD, charge-coupled device).

the beagle was observed clearly with sufficient luminous intensity. Color rendering in the red range was good, and the red areas on the gastric mucosa and blood vessels were observed in color tones that were satisfactory for endoscopic purposes (Figure 2 a, b).

The use of LEDs may enable the miniaturization of the whole endoscope system and remove constraints on the design of its shape. In contrast to the capsule type, the LED-illuminated endoscope is expected to retain all the functions of the conventional endoscope such as operability and biopsy [5]. We believe the new LEDilluminated endoscope will help us to perform routine gastrointestinal endoscopy safely and effectively.

\section{Acknowledgment}

Supported by a grant from the Intelligent Cluster Project of the Ministry of Education, Culture, Sports, Science and Technology, Japan.

Competing interests: None
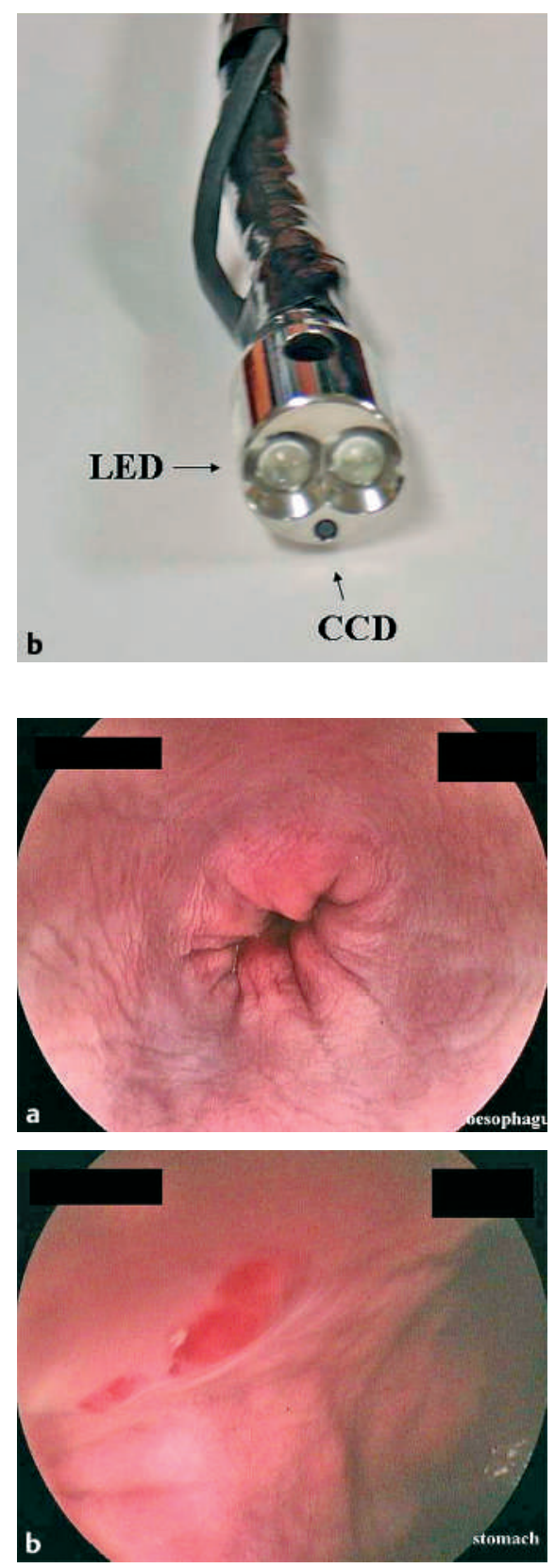

Figure 2 In vivo endoscopic images from a beagle, using the new white LED illumination: $\mathbf{a}$ the esophagus, and $\mathbf{b}$ the red areas of the gastric mucosa. 
H. Yanai', K. Okita², K. Kikuchi'

K. Tadatomo ${ }^{4}$, H. Nakamura ${ }^{2}$,

J. Nishikawa ${ }^{2}$, T. Yoshida ${ }^{2}$, T. Taguchi ${ }^{4}$

${ }^{1}$ Department of Gastroenterology and Hepatology, National Hospital Organization Kanmon Medical Center, Shimonoseki, Japan

2 Department of Gastroenterology and Hepatology, Yamaguchi University School of Medicine, Ube, Japan

${ }^{3}$ Fujinon Corporation, Saitama, Japan

${ }^{4}$ Department of Electrical and Electronic Engineering, Faculty of Engineering, Yamaguchi University, Ube, Japan

\section{References}

${ }^{1}$ Schuman BM. Upper gastrointestinal endoscopy. In: Haubrich WS, Schaffner F, Berk JE (eds). Bockus Gastroenterology. Philadelphia: W. B. Saunders 1995: $295-309$

2 Pennazio M, Santucci R, Rondonotti Eet al. Outcome of patients with obscure gastrointestinal bleeding after capsule endoscopy: report of 100 consecutive cases. Gastroenterology 2004; 126: 643-653

${ }^{3}$ Nakamura S, Fasol G. The blue laser diode. Berlin: Springer-Verlag 1997: 216-221

${ }^{4}$ Taguchi T. Present status of white LED lighting technology in Japan. J Light Vis Env 2003; 27: $1-6$

5 Yanai H, Nakamura H, Okita K. Development of the white light-emitting diodeilluminated digestive endoscope [abstract]. Gastrointest Endosc 2004; 59: AB145

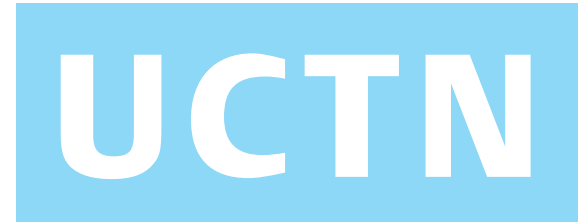

Corresponding Author

H. Yanai, M.D.

Department of Gastroenterology and Hepatology

National Hospital Organization Kanmon

Medical Center

Ushiroda 1-1-1

Shimonoseki

Yamaguchi 751-8501

Japan

Fax: $\quad+81-832-34-1416$

Phone: +81-832-22-6216

E-mail: yanaih@simonoseki2.hosp.go.jp 